Dhaka Univ. J. Biol. Sco. 20(2): 123-130, 2011 (July)

\title{
DRUG RESISTANCE AND CURLI FIMBRINATION OF ESCHERICHIA COLI ISOLATED FROM BANGLADESHI PATIENTS WITH URINARY TRACT INFECTIONS
}

\author{
M. Murshida Mahbub, Nafisa Azmuda, Belal Maumood, Sirajul Islam Khan, \\ Nils-KÅRe BirKeland ${ }^{1}$ AND Humaira AKHTER* \\ Department of Microbiology, University of Dhaka, Dhaka-1000, Bangladesh
}

Key words: Escherichia coli, Urinary tract infection, Antimicrobial resistance, Curli fimbriae

\begin{abstract}
A total of 27 isolates from patients with urinary tract infection (UTI) were screened and of these 12 were found to be Escherichia coli. All the E. coli isolates were multidrug resistant. Among the antibiotics, imipenem and polymyxin B were found to be the best, while oxacillin, cefsulodine and methicillin proved to be worst in effectiveness against the studied E. coli isolates. Only $42 \%$ of the E. coli were susceptible to trimethoprim-sulfamethoxazole (TMP-SMX), the current drug of choice for treating UTI. Middle ranged plasmids were observed in the studied isolates. Five strains expressed curli fimbriae and two elaborated cellulose; two other strains produced both curli and cellulose as extracellular matrix component.
\end{abstract}

\section{Introduction}

Community-acquired urinary tract infections (UTIs) are among the most common bacterial infections especially in women. Uropathogenic Escherichia coli (UPEC) strains are responsible for the majority of uncomplicated urinary tract infections. ${ }^{(1)}$ The reasons for the geographic variations in prevalence of antibiotic resistance among E. coli causing urinary tract infections are poorly understood.(2) Therapy for these infections is usually begun before results of microbiological tests are known. Furthermore, in women with acute uncomplicated cystitis, empirical therapy without a pre-therapy urine culture is often used. Consequent to this, antimicrobial resistance among uropathogens causing community-acquired UTIs, both cystitis and pyelonephritis, is increasing.

Plasmid borne resistance profile has been a major issue because this extrachromosomal genetic material plays a role in the spread of antimicrobial drug resistance. But the molecular epidemiologic study of these diverse and mobile elements is complex because of their replication system which dictates plasmid's behavior such as host range and copy number. ${ }^{(3)}$

UTI strains have been found to have the highest frequency of mutator strains. Mutator strains, having a defective mismatch repair system, are prone to high mutation

${ }^{*}$ Corresponding author. ${ }^{1}$ Department of Biology, University of Bergen, Bergen 7800 5020, Norway. 
rates. ${ }^{(4)}$ Because most of these isolates are pathogens, it has been hypothesized that mutator and hyper recombination phenotypes may accelerate the evolution of pathogenic strains by, e.g., increasing the variation of surface antigens, as well as by facilitating the acquisition of pathogenic determinants and antibiotic resistance. It has been observed that the levels of resistance to antibiotics were significantly higher in mutator than in nonmutator pathogenic $P$. aeruginosa isolates. ${ }^{(5)}$

Adhesion to abiotic surfaces (biofilm formation) and expression of curli fimbriae and cellulose as extracellular matrix components is an important property for uropathogens. Curli fimbriae and/or cellulose are expressed by E. coli, Salmonella spp. and other Enterobacteriaceae. ${ }^{(6-7)}$ While the contribution of cellulose to virulence is not well-known, several virulence-associated features have been assigned to curli fimbriae. ${ }^{(8-9)}$

The present study was conducted to determine antibiotic resistance profile of urine isolates of $E$. coli from urinary tract infected patients; plasmid content of the isolates were also examined to decipher whether their presence correlated with the antibiogram. The current investigation also had a goal to seek the potential of the UPEC isolates to express curli and/or cellulose as extracellular material.

\section{Materials and Methods}

Urine samples of patients with urinary tract infection were collected from a hospital in Sylhet, Bangladesh. Samples were streaked on nutrient agar and then selected on MacConkey agar. Colonies that presumptively matched with the appearance of E. coli on MacConkey agar were subcultured on nutrient agar. The clinical isolates were then stabbed into $\mathrm{T}_{1} \mathrm{~N}_{1}$ soft agar and transported into the laboratory.

The isolates were resuscitated in nutrient agar and given laboratory codes as identification numbers. The organisms were then identified as E. coli following standard microbiological and biochemical methods. ${ }^{(10)}$

The susceptibility tests of all the 12 isolates to the antimicrobial agents were measured in vitro by the modified Kirby-Bauer disk diffusion method.(11) Commercially available antimicrobial discs (Oxoid, In Vitro Diagnosticum) used in this study were Polymyxin B $(30 \mu \mathrm{g})$, Spectinomycin $(100 \mu \mathrm{g})$, Oxacillin $(1 \mu \mathrm{g})$, Ciprofloxacin $(5 \mu \mathrm{g})$, Kanamycin $(30 \mu \mathrm{g})$, Sulfomethoxazole/trimethoprim $(25 \mu \mathrm{g})$, Cephalexin $(30 \mu \mathrm{g})$, Nalidixic acid $(30 \mu \mathrm{g})$, Cefsulodine $(30 \mu \mathrm{g})$, Ceftizidime $(30 \mu \mathrm{g})$, Rifampicin $(30 \mu \mathrm{g})$, Ampicillin $(10 \mu \mathrm{g})$, Methicillin $(5 \mu \mathrm{g})$, Cefotaxim $(30 \mu \mathrm{g})$, Imipenem $(10 \mu \mathrm{g})$, and Ceftriaxone $(30 \mu \mathrm{g})$. E. coli ATCC 25922 was used as control strain for susceptibility studies.

Along with scoring the clear zone for antibiotic susceptibility the strains were also checked for colonies growing inside the growth inhibition zone (squatter colonies).(4) The

presence of squatter colonies reflects the high frequency of mutations conferring resistance to antibiotics. 
Plasmid DNA was extracted according to the procedure as described by Birnboim and Doly(12) with slight modification. The isolated plasmid DNA thus prepared was electrophoresed in agarose gel followed by staining for $10 \mathrm{~min}$ in ethidium bromide (0.5 $\mu \mathrm{g} / \mathrm{ml}$ ) solution at room temperature. DNA bands were visualized and photographed using UV transilluminator (Gel Doc, Bio-Rad, USA). The molecular weight of the unknown plasmid DNA was determined on the basis of its mobility through agarose gel and was compared with the mobility of the known molecular weight plasmids $(1 \mathrm{~kb}$ supercoiled plasmid marker, Invitrogen).

To examine whether the studied isolates express their genes for formation of curli fimbriae, the cells were inoculated on Congo red (CR) medium (composition: 1\% casamino acids, $0.15 \%$ yeast extract, $0.005 \% \mathrm{MgSO}_{4}$ and $2 \%$ agar with $0.004 \%$ congo red plus $0.001 \%$ coomassie blue). ${ }^{(13-14)}$ After incubation at $28^{\circ} \mathrm{C}$ for $48 \mathrm{hrs}$ colony morphology was inspected on CR plates. Colony morphologies on CR plates were scored according to the basic morphotypes ${ }^{(15)}$ previously detected in S. typhimurium: these were categorized as, rdar (violet colony, expresses curli fimbriae and cellulose), pdar (pink colony, expresses cellulose), bdar (brown colony, expresses curli fimbriae) and saw (no expression of curli fimbriae or cellulose).

\section{Results and Discussion}

High potential for developing drug resistance among pathogenic isolates of $E$. coli has been reported earlier.(3) The clinical isolates of $E$. coli are continuously exposed to the hospital environment where they acquire resistance to numerous antibiotics. Resistance properties accrue through different routes, such as natural or intrinsic resistance (inaccessibility of the target, multidrug efflux systems and drug inactivation), mutational change, and attainment of extra-chromosomal materials. Thus intensive analysis of several factors especially molecular characterization is required.

In the present study, 12 strains were identified as E. coli from 27 urine specimens collected from urinary tract infected patients. Of the 12 isolates, all were found to be resistant to oxacillin, cefsulodine and methicillin. Trimethoprim-sulfamethoxazole (TMPSMX), the current drug of choice ${ }^{(16)}$ for treatment of acute uncomplicated cystitis in women was found to be effective against only $42 \%$ of the isolates. The most successful drugs appeared in this study were polymyxin B and imipenem (sensitivity 100\%) (Fig.1). Ceftazidime, cefotaxim and ceftriaxone were found to be the next most efficient drug in this investigation. All of the studied isolates were found to be multidrug resistant. Among the isolates, MCL 15, 27, 28, 29 and 30 were resistant to $\geq 10$ of the 15 antibiotics (Table 1).

Almost all of the strains contained plasmids of mid-range sizes (Fig. 2) which could be easily transferred between bacterial floras; if such transferrable elements carry the resistance (R) factor, drug resistance can spread quickly. However, we found no 
correlation between the presence of plasmids and the number of antibiotic resistance among the isolates. Plasmid curing from the resistance isolates could be done in future to track whether the antibiotic resistance property was retained or not in the cured strain. Additionally, each plasmid could be transferred in a plasmid-free bacterium to see whether the transformed bacterium shows resistance against any specific antibiotic or not.

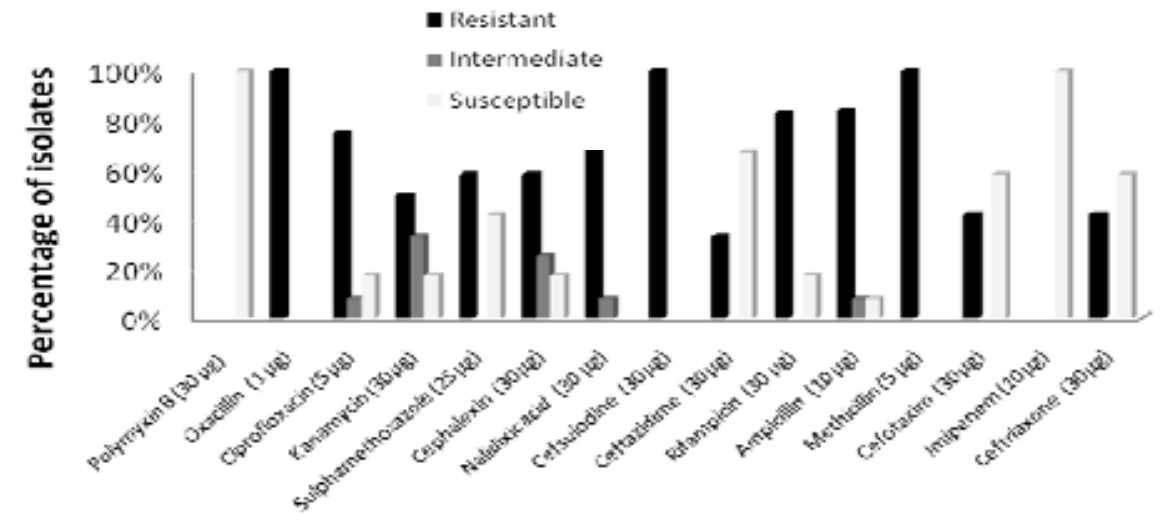

Antibiotics

Fig. 1. Antimicrobial resistance of the studied UPEC isolates.

Table 1. Antibiotic susceptibility and presence of squatter colonies in inhibition zone.

\begin{tabular}{lcccc}
\hline Isolate & \multicolumn{3}{c}{ No. of antibiotics } & $\begin{array}{c}\text { Squatter colonies found in } \\
\text { inhibition zones }\end{array}$ \\
\cline { 2 - 4 } & Resistant to & Intermediate to & Sensitive to & CL, RD \\
MCL 2B & 7 & 2 & 6 & CL, RD, AMP, CAZ, CTX \\
MCL 4A & 5 & 2 & 8 & SH, CFS \\
MCL 7 & 7 & 0 & 8 & CFS, AMP \\
MCL 10B & 8 & 1 & 6 & CL, CFS, CRO \\
MCL 13 & 9 & 1 & 5 & None \\
MCL 14 & 8 & 2 & 5 & CAZ \\
MCL 15 & 13 & 0 & 2 & CTX \\
MCL 26B & 7 & 1 & 7 & CTX \\
MCL 27 & 11 & 0 & 4 & None \\
MCL 28 & 13 & 0 & 2 & None \\
MCL 29 & 10 & 1 & 4 & None \\
MCL 30 & 12 & 0 & 3 & \\
\hline
\end{tabular}

Presence of squatter colonies inside the growth inhibition zone (around fosfomycin or rifampicin disc) has been used to differentiate between mutator and nonmutator strains. ${ }^{(17)}$ Denamur ${ }^{(4)}$ reported the exhibition of colonies inside the growth inhibition 
zone (squatter colonies) by the majority of mutator strains, but not by nonmutator strains. However the same authors ${ }^{(17-18)}$ reported the absence of the correlation between high mutation rates and antibiotic resistance; they explained this by the dynamics of selection and counter-selection of mutator alleles. In our study we observed squatter colonies inside the growth inhibition zone around different antibiotic discs in cases where isolates were resistant to lower number of antibiotics (Table 1).

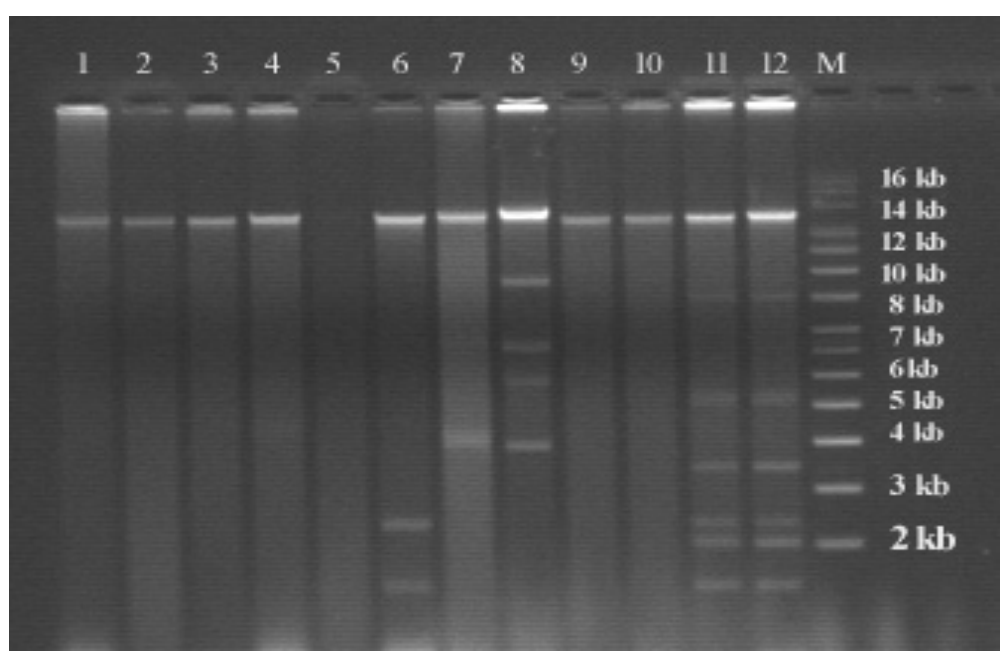

Fig. 2. Agarose gel electrophoresis of plasmid DNA from the studied uropathogenic E. coli isolates. Lane: M, supercoiled plasmid marker; 1, MCL 2B; 2, MCL 4A; 3, MCL 7; 4, MCL 10B; 5, MCL 13; 6, MCL 14; 7, MCL 15; 8, MCL 26B; 9, MCL 27; 10, MCL 28; 11, MCL 29; 12, MCL 30.

In the setting of urinary tract with many unfavorable factors along with frequent urine flushing, colonization and long-term persistence are advantageous to the uropathogenic organisms. Extracellular matrix components play important role in such ability of these bacteria. Adhesins can contribute to virulence by promotion of colonization, invasion and replication within uroepithelial cells. Tiba et al. (19) reported that the virulent strains of UPEC that cause cystitis typically produce, at least, one adhesion system.

We investigated curli and/or cellulose expression and observed that nine out of 12 $(75 \%)$ isolates expressed at least one of the biofilm extracellular matrix components (Table 2). The other three isolates, which were negative for expression in both components might possess systems for other adhesions or might not have expressed these components under the present experimental condition. When considered individually, seven of the 12 expressed curli fimbriae and four of the 12 elaborated cellulose as the extracellular material. Beforehand, cellulose was not known to contribute to the pathogenic behavior; but curli fimbriae have been ascribed to have relation with different 
virulent-associated features. However, a concrete idea about the role of curli fimbriae in pathogenicity is still not clear.(15)

Table 2. Expression of curli fimbriae in uropathogenic E. coli isolated from UTI patients.

\begin{tabular}{llcc}
\hline Isolate & Color (morphotype) & \multicolumn{2}{c}{ Expression } \\
\cline { 2 - 4 } & & Curli & Cellulose \\
\hline MCL 2B & Violet (rdar) & + & + \\
MCL 4A & Brown (bdar) & + & - \\
MCL 7 & Pink (pdar) & - & + \\
MCL 10B & Grayish white (saw) & - & - \\
MCL 13 & Brown (bdar) & + & - \\
MCL 14 & Brown (bdar) & + & - \\
MCL 15 & Violet (rdar) & + & + \\
MCL 26B & Brown (bdar) & + & - \\
MCL 27 & Brown (bdar) & + & - \\
MCL 28 & Pink (pdar) & - & + \\
MCL 29 & Grayish white (saw) & - & - \\
MCL 30 & Grayish white (saw) & - & - \\
\hline
\end{tabular}

Only two antibiotics were found effective, while the rest were found to be ineffective under the present study condition. The abuse of antibiotics may be responsible for the occurrence of drug resistance trait among these pathogens. The squatter colonies may be of interest in determining which mutation contributed to the drug resistance property. The expression of curli and/or cellulose is also of interest in elucidating the mechanism of biofilm formation which increases the capacity of the organisms to survive in a hostile environment.

\section{Acknowledgements}

The research was a joint collaboration between the Department of Microbiology, University of Dhaka, Bangladesh and the Department of Biology, Bergen University, Norway. The authors are grateful to NUFU, Norway for the funding of this project.

\section{References}

1. Lloyd AL, DA Rasko and HLT Mobley 2007. Defining genomic islands and uropathogenspecific genes in uropathogenic Escherichia coli. J. Bacteriol. 189(9): 3532-3546.

2. Gupta K, TM Hooton and WE Stamm 2001. Increasing antimicrobial resistance and the management of uncomplicated community-acquired urinary tract infections. Ann. Intern. Med. 135: 41-50. 
3. Jan N, SU Meshram and A Kulkarni 2009. Plasmid profile analysis of multidrug resistant E. coli isolated from UTI patients of Nagpur city, India. Rom. Biotechnol. Lett. 14(5): 4635-4640.

4. Denamur E, S Bonacorsi, A Giraud, P Duriez, F Hilali, C Amorin, E Bingen, A Andremont, B Picard, F Taddei and I Matic 2002. High Frequency of Mutator Strains among Human Uropathogenic Escherichia coli Isolates. J. Bacteriol. 184(2): 605-609.

5. Oliver A, R Canton, P Campo, F Baquero, and J Blazquez 2000. High frequency of hypermutable Pseudomonas aeruginosa in cystic fibrosis lung infection. Science 288:12511254.

6. Römling U, W Bokranz, W Rabsch, X Zogaj, M Nimtz and H Tschäpe 2003. Occurrence and regulation of the multicellular morphotype in Salmonella serovars important in human disease. Int. J. Med. Microbiol. 293: 273-285.

7. Zogaj X, W Bokranz, M Nimtz and U Römling 2003. Production of cellulose and curli fimbriae by members of the family Enterobacteriaceae isolated from the human gastrointestinal tract. Infect. Immun. 71: 4151-4158.

8. Olsen A, H Herwald, M Wikstrom, K Persson, E Mattsson and L Bjorck 2002. Identification of two protein-binding and functional regions of curli, a surface organelle and virulence determinant of Escherichia coli. J. Biol. Chem. 277: 34568-34572.

9. Sjöbring U, G Pohl and A Olsen 1994. Plasminogen, absorbed by Escherichia coli expressing curli or by Salmonella enteritidis expressing thin aggregative fimbriae, can be activated by simultaneously captured tissue-type plasminogen activator (t-PA). Mol. Microbiol. 14: 443-452.

10. World Health Organization 1987. Programme for control of diarrhoeal disease. pp. 9-20. In Manual for laboratory investigation of acute enteric infections. CDD/93.3, Rev. 1. World Health Organization, Geneva, Switzerland.

11. Bauer AW, WMM Kirby, JC Sherris and M Turk 1966. Antibiotic susceptibility testing by a standardized single disk method. Am. J. Clin. Pathol. 45: 493-496.

12. Birnboim HC and J Doly 1979. A rapid alkaline extraction procedure for screening recombinant plasmid DNA. Nucl. Acid. Res. 7:1513-1523.

13. Castonguay M, S van der Schaaf, W Koester, J Krooneman, $\mathrm{W}$ van der Meer, H Harmsen, $\mathrm{P}$ Landini 2006. Biofilm formation by Escherichia coli is stimulated by synergistic interactions and co-adhesion mechanisms with adherence-proficient bacteria. Res. Microbiol. 157: 471478.

14. Römling U, MRohde, A Olsen, S Normark and J Reinköster 2000. AgfD, the checkpoint of multicellular and aggregative behaviour in Salmonella typhimurium regulates at least two independent pathways. Mol. Microbiol. 36: 10-23.

15. Bokranz W, Wang X, Tschäpe $H$ and U Römling 2005. Expression of cellulose and curli fimbriae by Escherichia coli isolated from the gastrointestinal tract. J. Med. Microbiol. 54: 1171-1182.

16. Zhanel GG, JA Karlowsky, GKM Harding, A Carrie, T Mazzulli, DE Low, The Canadian Urinary Isolate Study Group and DJ Hoban 2000. A Canadian national surveillance study of urinary tract isolates from outpatients: comparison of the activities of trimethoprimsulfamethoxazole, ampicillin, mecillinam, nitrofurantoin, and ciprofloxacin. Antimicrob. Agents Chemother. 44: 1089-1092.

17. Denamur E and I Matic 2006. Evolution of mutation rates in bacteria. Mol. Microbiol. 60(4): 820-827. 
18. Denamur E, O Tenaillon, C Deschamps, D Skurnik, E Ronco, JL Gaillard 2005. Intermediate mutation frequencies favor evolution of multidrug resistance. Genetics 171: 825-827.

19. Tiba MR, T Yano, and DS Leite 2008. Genotypic characterization of virulence factors in Escherichia coli strains from patients with cystitis. Rev. Inst. Med. trop. S. Paulo, 50(5): 255-260.

(Manuscript received on 23 December, 2009; revised on 8 November, 2010) 